\title{
Superconducting gap and pseudogap
}

\author{
Guy Deutscher \\ School of Physics and Astronomy, Tel Aviv University \\ Ramat Aviv, Tel Aviv 69978, Israel \\ E-mail: guyde@post.tau.ac.il
}

Received November 22, 2005

\begin{abstract}
The discovery of the pseudogap has been a fundamental advance in uncovering the new physics of the high- $T_{c}$ cuprates. Yet, its meaning is still far from being clear. In particular, its relation to the superconducting gap remains an object of controversy. While many authors consider that it is a high temperature precursor of superconductivity, which turns into the superconducting gap at low temperatures, others contend that it is a normal state property related only indirectly to superconductivity. We review a number of experiments such as single particle tunneling, Andreev-Saint-James reflections and others, and conclude that in the underdoped regime there exists considerable evidence for the existence of two distinct energy scales, the superconducting gap and the pseudogap, which appear to merge into one another in overdoped samples.
\end{abstract}

PACS: 74.72.-h

Keywords: superconducting gap, pseudogap, tunneling spectroscopy.

\section{Introduction}

When a new superconductor is discovered, one of the primary objectives of research is to determine the value of its energy gap. This has proved to be a difficult task in the case of the cuprates. Twenty years after their discovery, no consensus on the superconducting energy gap has yet been reached. Somehow, the discovery of the pseudogap may have pushed this important question to the sidelines. Some of the main spectroscopic methods, tunneling and angle resolved photoemission spectroscopy (ARPES) have given somewhat strange results, particularly in underdoped samples where an energy gap is seen to increase as the critical temperature decreases, and to persist above the critical temperature. Originally discovered by NMR measurements which showed in underdoped samples a decrease of the spin density of states well above the critical temperature [1], the existence of a reduced density of states near the Fermi level in the normal state is at the origin of this behavior [2]. It extends up to an energy $\Delta_{p}$, called the pseudogap, directly related to STM and ARPES gap values measured at low temperatures.

In a pioneering work, Renner et al. [3] performed scanning tunneling spectroscopy (STM) measurements on $\mathrm{BiSrCaCuO}$ single crystals of various doping levels, and directly observed, particularly in underdoped samples, a depressed conductance above $T_{c}$ up to an energy scale that turned at low temperatures into a superconducting like-gap structure with a clear conductance peak. They proposed that the pseudogap seen above the critical temperature is a precursor of the superconducting gap, or in other terms that a pairing amplitude exists in these samples well into the normal state. A possible theoretical framework that would fit these observations could be a BCS to Bose-Einstein (BE) crossover, with pairs forming above $T_{C}$ in the underdoped regime due to a strong interaction. $2 \Delta_{p}$ would be the energy required to break a pair, below or above $T_{c}$. The measured large values of the ratio $\Delta_{p} / T_{c}$ would reflect the role played by thermal fluctuations, coherence being established by a Kosterlitz-Thouless transition. The general idea that the transition to superconductivity is of that type is still that of leading theorists [4], who have developed models where it is not the electron-phonon mechanism that is at the origin of superconductivity.

In this contribution we wish to point out that a number of experimental results do not necessarily agree with this description. Our main point is that tunneling or ARPES are excellent tools to study the pseudogap, but that they leave open the question of 
what the superconducting gap really is. A better way to determine the latter is to study Andreev-Saint-James (ASJ) reflections, which are uniquely sensitive to the superconducting order parameter [5]. By comparing STM and ASJ spectroscopy measurements, we reach the conclusion that in underdoped samples they give two distinct energy scales. The lower one, detected by ASJ reflections, is the superconducting gap and the higher one seen by STM is the pseudogap, the two scales becoming progressively distinct as one goes further into the underdoped regime. Other recent experimental results such as the doping dependence of the superfluid density and of the $\mu \mathrm{SR}$ lineshape and fluctuation effects in Josephson junctions support the conclusion of two distinct energy scales in the underdoped regime.

\section{Single particle tunneling versus Andreev- Saint-James spectroscopy}

Single particle tunneling has been the method of choice to measure the superconducting energy gap in low-temperature superconductors (LTS), and more generally to obtain their detailed density of states (DOS) [6]. Impressing fits between theory and experiment allowed a precise determination of the value of the gap. One of the main features of a superconductivity density of states is the occurrence of a coherence peak at the gap edge, which allows one to distinguish it in a straightforward way from a semiconducting gap. In an $s$-wave superconductor, the density of states diverges at the gap edge as $1 /(E-\Delta)^{1 / 2}$, while in a $d$-wave superconductor the divergence is only logarithmic. In the former case the DOS is zero up to the gap, while in the latter one it increases linearly from the Fermi energy.

Although in its broad lines the STM conductance data of Renner et al. resembles that of a $d$-wave superconductor, there are substantial differences that prevent fitting the data to a $d$-wave DOS, and to obtain from that fit the value of the energy gap. Principally, the conductance peak is much broader than the coherence peak at the gap edge should be, and it is not clear that the conductance at small bias increases linearly. Moreover, the conductance peak becomes broader and weaker as underdoping is increased. Also, the position of the conductance peak can vary substantially from place to place on a given sample, which is a little bit surprising for a superconducting gap in samples supposed to be uniform. Gap values determined by ARPES (which also detects single particle excitations [7]) on $\mathrm{BiSrCaCuO}$ follow the same trend as the tunneling gap. Samples near optimum doping can also show gap values which vary from sample to sample in a rather broad range (typically 25 to $40 \mathrm{meV}$ ).

ASJ reflections [5] provide an alternative way to determine the value of the superconducting gap. Electron scattering at a normal metal $(\mathrm{N}) /$ superconductor $(\mathrm{S})$ interface includes a process in which an incoming electron from the $N$ side is reflected back as a hole of opposite momentum, while a Cooper pair flows on the $\mathrm{S}$ side. For a clean transparent interface, with similar Fermi velocities on both sides, this is the dominant scattering process. It leads to an enhancement of the conductance of the contact, (ideally by a factor of 2) below the gap, compared to its normal state value. At higher energies, excitations in $\mathrm{S}$ return to their normal state character and the conductance goes back to its normal state value. The change in conductance allows one to identify the gap edge. In a BCS superconductor, ASJ reflections and single particle tunneling give the same gap value. The shape of the characteristics changes progressively from ASJ to tunneling when the interface transparency is reduced [8].

ASJ reflections at $\mathrm{N} /$ high- $T_{c}$ interfaces were first observed by point contact (PC) between a gold tip and optimally doped single crystal quality YBCO [5]. An enhanced conductance at low bias was clearly observed, as well as a well defined gap edge at $20 \mathrm{meV}$. This value was in excellent agreement with that determined by STM by Fischer et al. on optimally doped YBCO single crystal [9]. By contrast, PC measurements on $\mathrm{BiSrCaCuO}$ samples showed that ASJ reflections occur over an energy scale $\Delta_{c}$ smaller than the STM conductance peak position $\Delta_{p}$, particularly for underdoped samples. This result contradicts the proposed identification of the tunneling gap with the superconducting gap at all doping levels.

The contrast between single particle tunneling and ASJ spectroscopy is best seen by comparing directly the respective data. We show Fig. 1 and Fig. 2 point contact ASJ and STM characteristics on the $\mathrm{BiO}$ surface of $\mathrm{BiSrCaCuO}$ single crystals [10]. STM measurements were performed on a cleaved sample while PC measurements were in-plane. The two methods should in principle be equivalent since STM measures the in-plane DOS averaged over all directions. The PC data could be fitted very well with the theory of Kashiwaya and Tanaka (KT) [11] assuming a $d$-wave order parameter, with a gap of about $20 \mathrm{meV}$, a small barrier parameter $Z$, and only a small broadening parameter $\Gamma$. Note also in Fig. 1 the clean triangular shape of the characteristic near zero bias, a feature predicted by KT for an order parameter having the $d$-wave symmetry. The series of STM characteristics were acquired on different locations of one sample. In 


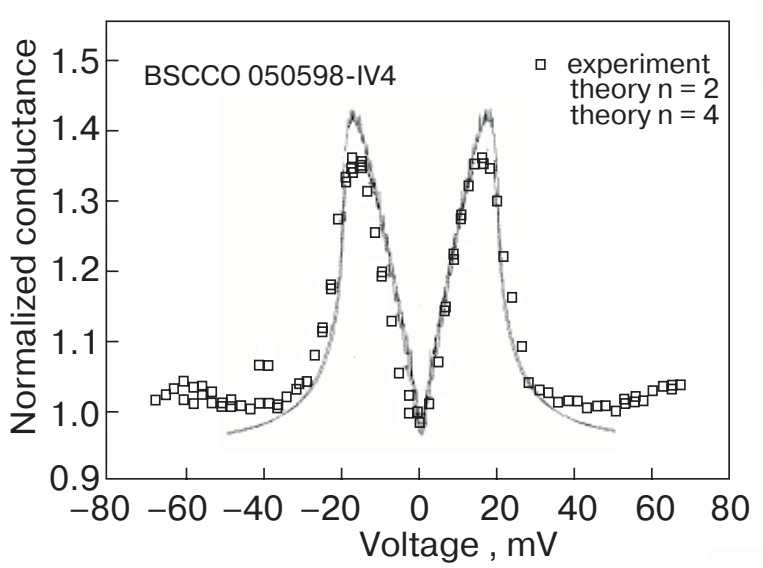

Fig. 1. Conductance characteristic of a contact between a gold point and a $\mathrm{BiSrCaCuO}$ in-plane oriented sample (after A. Kohen and I. D'Gourno, adapted from Ref. 5). Note the clear $V$ shape of the characteristic at low bias, in agreement with the predictions of Ref. 10 for a $d$-wave symmetry. The full line is the theoretical fit to the data. $\left(Z=0.8, \Delta=\Delta_{0} \cos (n \theta), \Delta_{0}=20 \mathrm{meV}\right)$.

most of them one can observe a hump around 20 to $25 \mathrm{meV}$ (corresponding approximately to the PC conductance peak) below which the characteristics fall on top of each other, followed by a broad peak structure whose position varies considerably from place to place. It is clear that it is not possible in general to fit these characteristics with a $d$-wave density of states or any other known symmetry with the possible exception of the sharpest characteristic which has a well developed peak at $25 \mathrm{meV}$. Such

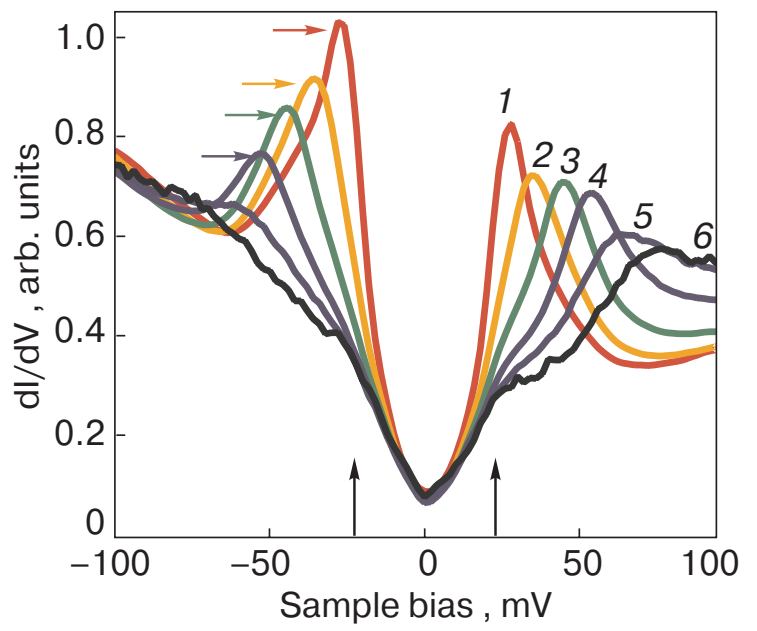

Fig. 2. STM characteristics measured at various locations on a cleaved $\mathrm{BiSrCaCuO}$ single crystal. Note that the location of the peak in the sharpest characteristic is close to that of the peak in the conductance in Fig. 1 (after Ref. 10). The characteristic at low bias is more rounded than that shown Fig. 1.

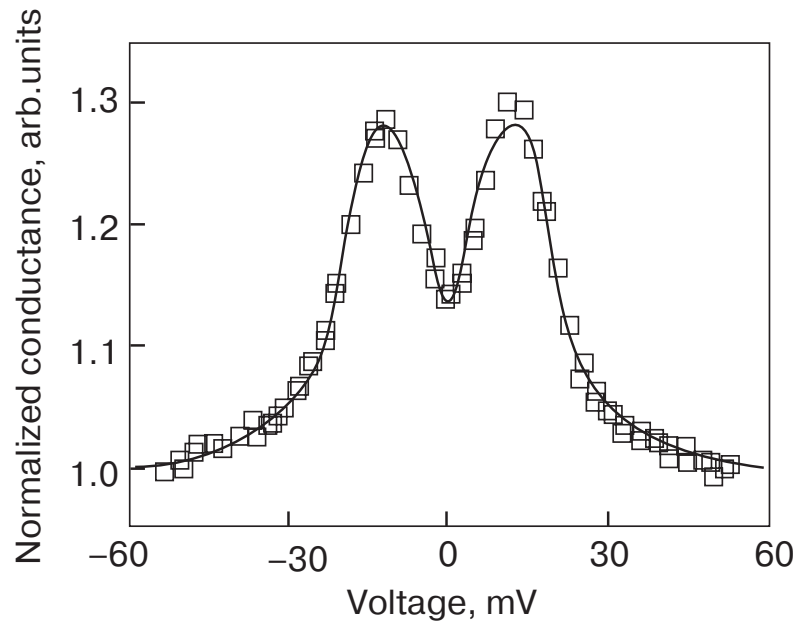

Fig. 3. Conductance of a contact between a gold tip and an in-plane oriented YBCO film. The $V$ shape at low bias is similar to that seen in Fig. 1.

characteristics are reported to be more often seen in overdoped samples. The enhanced conductance in the PC data occurs in the low bias range where STM data are uniform, indicating that the low bias DOS is directly related to superconductivity. But the high bias part of most STM characteristics and in particular the broad conductance peaks occur in a bias range far beyond that where the effect of ASJ reflections is observed. It is therefore doubtful that the pseudogap, identified with the broad peaks, is directly related to superconductivity. This is in line with the observation that ASJ reflections are never seen above $T_{c}$, even in underdoped samples having a large pseudogap [12]. If the pseudogap was a precursor of superconductivity, one would expect to see some trace of ASJ reflections above the critical temperature.

From the comparison between STM and PC data, we conclude that the homogeneous low energy part of the STM data may reflect the superconducting DOS, while the inhomogeneous high energy part does not. In any case, it is not possible to extract from STM data a value of the superconducting energy gap, particularly for underdoped samples. By contrast, PC - ASJ data can be fitted extremely well to theory and give an unambiguous value for the gap. We have obtained characteristics similar to that shown Fig. 1 in a large number of samples. As an additional example we show Fig. 3 in-plane PC data on an YBCO film. The fit quality is as good as that obtained for $\mathrm{BiSrCaCuO}$. In particular, the $V$ shape at low bias is very clean.

The observation of ASJ reflections below the tunneling pseudogap raises however some theoretical difficulties. Nozieres and Pistolesi have shown that no ASJ enhanced conductance below that gap is ex- 
pected, whether it is a semiconducting one or a strong coupling gap [13]. As we have remarked somewhere else [5] this difficulty can be removed if there remains a sufficient density of states in the normal state within the pseudogap. Recent theoretical work does give us some idea of the normal state density of states in the pseudogap regime. Kyung et al. [14] have performed a Monte Carlo calculation of the normal state DOS at zero temperature in the repulsive Hubbard model, as a function of doping, and have come up with results that show indeed a substantial DOS within the pseudogap. This immediately explains why ASJ reflections can indeed be observed below the tunneling gap.

\section{Structure of the superconducting tunneling DOS}

If the pseudogap is really a normal state property not directly related to superconductivity, the tunneling conductance should be of the form:

$$
(d I / d V)_{S}=(d I / d V)_{N} N_{S}(E)
$$

where $(d I / d V)_{S}$ and $(d I / d V)_{N}$ are respectively the superconducting state and normal state conductances, and $\mathrm{N}_{S}(E)$ is the superconducting density of states.

In the LTS, one can safely assume that $(d I / d V)_{N}$ is a constant within the small energy range of the gap (compared to the height of the barrier). If necessary, it can also be determined experimentally by quenching superconductivity with a magnetic field. In the HTS, none of this works: we have no right to assume that $(d I / d V)_{N}$ is constant, and we do not have at our disposal strong enough magnetic fields to quench altogether superconductivity. One cannot, therefore, retrieve directly $N_{S}(E)$ from tunneling data. One of the advantages of using ASJ reflections to determine the superconducting gap, is precisely that there are no such reflections in the normal state.

Although there are now, as said above, some normal state DOS calculations in the pseudogap regime [14], these are not still sufficiently detailed to be used to retrieve the superconducting density of states from experimental tunneling data. But, even if there are rapid changes of the normal state DOS in the region of the superconducting gap, there should be some remnant of the coherence peak at the superconducting gap edge. What we wish to show now is that there are indeed experimental indications for such a structure in tunneling characteristics.

In YBCO, Maggio-Aprile et al. [15] reported a double peak in their STM data, a narrower one at $20 \mathrm{meV}$ which fits well with ASJ data as mentioned above, and might indeed be the coherence peak, and a broader one around $30 \mathrm{meV}$, which may correspond to the pseudogap. Double conductance peaks were also

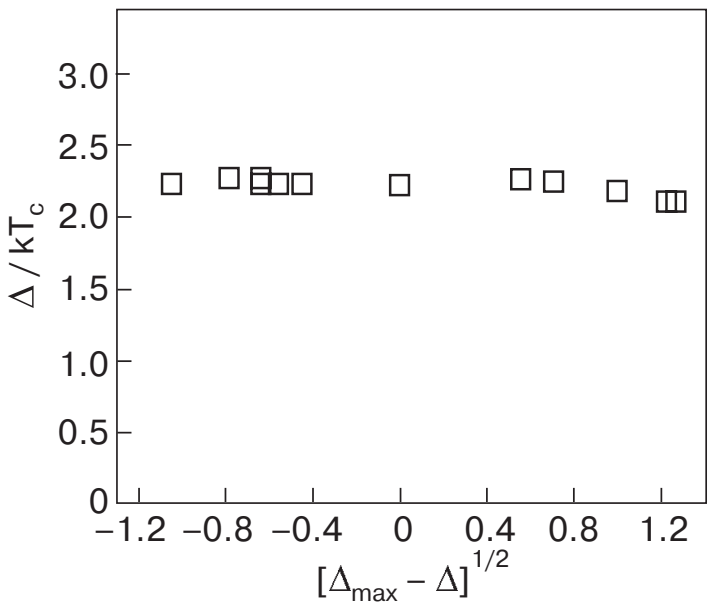

Fig. 4. Ratio of the ASJ gap to the critical temperature in YBCO films covering a range from underdoped to overdoped. The ratio is very close to the weak coupling limit predicted for a $d$-wave superconductor (after Ref.18).

reported by Racah on $\mathrm{YBCO} / \mathrm{In}$ junctions [16], and more recently by Vedeneev and Maude on slightly underdoped single crystal $\mathrm{BiSrCaCuO}$ break junctions [17]. In this latter work, there is a sharp peak at about $25 \mathrm{meV}$ and a broader one whose position varies considerably from one junction to another. Moreover, the narrow peak is washed out by a magnetic field, while the broad one is not. The position of the narrow peak corresponds exactly to that of the sharpest STM characteristic reported by McElroy et al. [10]. The hump in their STM data may be a remnant of the coherence peak.

There is therefore some experimental evidence for a narrow tunneling DOS peak, possibly a superconducting coherence peak, followed by a broader one, which characterizes the pseudogap.

If indeed the coherence peak occurs at the superconducting gap, it should follow the same doping dependence as the critical temperature, as the ASJ energy scale does [9]. Dagan et al. have performed tunneling experiments on (110) oriented YBCO films, for which characteristics are dominated by a zero bias conductance peak due to ASJ surface bound states, but show also a peak at a bias corresponding to the value of the superconducting gap, presumably because of surface roughness [18]. For samples going from overdoped to underdoped, the position of this peak was found to correlate extremely well with the critical temperature (Fig. 4). This observation is consistent with the variation with doping of the position of the narrow peak seen in STM data on YBCO [19]. 


\section{Additional experimental indications for a distinct superconducting energy scale}

Sonier et al. have determined the vortex core size in strongly underdoped YBCO single crystals from muon spin resonance [20]. They found that it increases as the oxygen doping is reduced, the increase being by about a factor of 2 between an optimum doped $\left(T_{c}=92 \mathrm{~K}\right)$ and a strongly underdoped $\left(T_{c}=55 \mathrm{~K}\right)$ sample. They concluded that quasiparticle and superconducting length scales are different in underdoped samples, the superconducting length scale being inversely proportional to the critical temperature. This conclusion is consistent with our view that there exists in the cuprates a coherence energy scale which has the same doping dependence as the critical temperature [9].

Zuev et al. [21] have measured the penetration depth in very underdoped YBCO films, and have concluded that the superfluid density is not proportional to the critical temperature as previously thought. This proportionality was one of the experimental results supporting the idea that the critical temperature in these samples is reduced by strong thermal fluctuations below the mean field temperature given by the pseudogap value. They found that in fact the characteristic length for the Kosterlitz-Thouless-Berezinski vortex unbinding transition is the film thickness rather than that of the $\mathrm{CuO}_{2}$ bilayers, which contradicts the view that the critical temperature is set by quasi-2D thermal fluctuations in these bilayers. In other terms, thermal fluctuations in severely underdoped films are similar to those in a BCS superconductor of similar thickness and normal state conductivity.

Bergeal [22] studied Josephson junctions prepared between optimally doped YBCO films and strongly underdoped ones $\left(T_{c}=60 \mathrm{~K}\right)$, in the temperature range between the two critical temperatures. The purpose of this experiment was to detect the large fluctuations expected in the case where preformed pairs exist in the underdoped film well above its critical temperature, which would manifest themselves by a conductance peak at low bias persisting up to the critical temperature of the optimally doped film. Bergeal did observe a conductance peak, but only in a limited range of temperatures of about 10 to $15 \mathrm{~K}$ above the critical temperature of the underdoped film. This is the same range where the effect of fluctuations is seen in the Josephson characteristics between two optimally doped films, above their critical temperature. The conclusion is that fluctuations of the order parameter in underdoped films are similar to those in optimally doped ones, and are well described as the usual Gaussian fluctuations above the critical temperature of a BCS superconductor.

We note however that the observation of a Nernst effect at high fields and high temperatures may be in contradiction with the above [22].

\section{Conclusions}

Precise values of the superconducting energy gap in the cuprates have been determined more accurately by probing Andreev - Saint-James reflections in point contacts rather than by tunneling experiments. The latter, as well as ARPES measurements, are better suited for studies of the pseudogap. Andreev-Saint-James reflections have revealed that the superconducting energy gap and the pseudogap are different in underdoped samples, the former varying with doping as the critical temperature does. There exists some recent evidence for the existence of a coherence peak at the superconducting energy gap in tunneling experiments, mostly in break-junctions, at an energy which is consistent with ASJ point contact data. The conclusion that the pseudogap is not directly related to superconductivity is supported by the doping dependence of the coherence length determined by muon spin rotation experiments, and by the lack of evidence for large thermal fluctuation effects in several experiments, with the possible exception of the observation of a Nernst effect at high fields and high temperatures.

This work was supported in part by the Israel Science Foundation, by the Heinrich Hertz - Minerva Center for High Temperature Superconductivity and by the Oren Family Chair for Experimental Solid State Physics.

1. H. Alloul, T. Ohno, and P. Mendels, Phys. Rev. Lett. 63, 1700 (1989).

2. T. Timusk and B. Statt, Rep. Prog. Phys. 62, 61 (1999).

3. C. Renner, B. Revaz, J.Y. Genoud, K. Kadowski, and O. Fischer, Phys. Rev. Lett. 80, 149 (1997).

4. P.W. Anderson, Fiz. Nizk. Temp. 32, 381 (2006).

5. For a review, see G. Deutscher, Rev. Mod. Phys. 77, 109 (2005).

6. J. Rowell and McMillan, in: Treatise on Superconductivity, R.D. Parks (ed.)

7. H. Ding et al., Nature 382, 51 (1996).

8. G.E. Blonder, M. Tinkham, and T.M. Klapwijk, Phys. Rev. B25, 4515 (1982).

9. G. Deutscher, Nature 397, 410 (1999).

10. K. McElroy et al., Phys. Rev. Lett. 94, 197005 (2005).

11. S. Kashiwaya and Y. Tanaka, Rep. Prog. Phys. 63, 1641 (2000)

12. Y. Dagan, R. Krupke, G. Deutscher, and A. Revcolevski, Phys. Rev. B61, 7012 (2000). 
13. P. Nozieres, quoted in Ref. 5, and private communication.

14. B. Kyung, S.S. Kancharla, D. Senecal, A.M. Tremblay, M. Civelli, and G. Kotliar, cond-mat/0502565.

15. L. Maggio-Aprile et al., Phys. Rev. Lett. 75, 2754 (1997).

16. D. Racah, PhD thesis Tel Aviv.

17. S.I. Vedeneev and D.K. Maude, cond-mat/0510707.
18. Y. Dagan and G. Deutscher, Europhys. Lett. 57, 444 (2002).

19. O. Fischer, private communication.

20. J.E. Sonier et al., cond-mat/05-8079.

21. Y. Zuev, Mun Seog Kim, and T.R. Lemberger, Phys. Rev. Lett. 95, 137002 (2005).

22. N. Bergeal, PhD thesis, Paris (2005). 